\title{
Methods of analysis of the power system security
}

\author{
Alexey Osak ${ }^{1, *}$, Daniil Panasetsky ${ }^{1}$, and Elena Buzina ${ }^{1}$ \\ ${ }^{1}$ ESI SB RAS, 664033 Lermontov str., 130, Irkutsk, Russia
}

\begin{abstract}
Increasing the intelligent level of the PS control systems caused by the implementation of Smart technologies changes the structure and the properties of PS and increases the importance of system reliability analysis. System reliability analysis includes two components - the balance analysis and the regime analysis. On the one hand, there are a large number of studies that assess the reliability of the power system examining various aspects and methods of solving this problem. In practice, the security analysis is limited by the calculations of power flows, static and dynamic stability for a number of forecast periods for the normal and repair circuits considering the most severe disturbances. The existing approach allows defining the requirements and adjusting emergency control systems, but does not allow evaluating and comparing solutions for power grid constructions. The authors propose a new method for power system reliability evaluation, which is suitable for planning development and operation of power systems. The method includes a general description of the algorithm which allows to compare various development scenarios, as well as to assess the reliability level of their implementation.
\end{abstract}

\section{Introduction}

The rise of intelligent level of the power system (PS) control due to the implementation of Smart technologies and conception of intelligent power system (IPS) of active-adaptive grid (AAG) changes the structure and characteristics of PS [1-2], simultaneously increasing the importance of assessment power system security, as aspect of system reliability. System reliability (PS reliability) includes balance and regime components.

In Russia, in accordance with [3], power system security is understood as a property of the system to maintain the prescribed modes of operation when conditions change, element failures and sudden perturbations occur. In other countries, the term security has a similar meaning, which is understood as the ability of the system to withstand sudden disturbances [4-6]. There is distinguish between static and dynamic power system regime security. In assessing static regime security, dynamic transient processes in PS are not taken into account, the model of the system is limited to the equations of steady-state. Dynamic regime security is related to the dynamics of PS behavior, when a nonlinear model of transient processes in PS or its linearized version can be considered.

There is a great number of studies on assessment of power system reliability (in particular [7-8]) dealing with various aspects and methods for solution of this problem. In practice, the security analysis is limited by calculations of static and dynamic stability for a number of forecast periods for normal and repair circuits considering the most severe disturbances [9]. Such an approach allows us to define requirements and adjust emergency control (EC). However, it cannot assess and compare solutions for power grid constructions.

The authors propose a new method to determine application of various aspects of security at different stages of development planning and during operation power system. A technique and an algorithm are proposed that allow to determine the magnitude of possible under-supply of electricity for various emergency situations, taking into account the emergency control and the expected actions of the dispatcher. This approach allows us to determine the mathematical expectation of annual damage from all accidents in PS, resulting in a shortage of electricity.

The authors propose the new method of steady state calculation of PS taking into account the discrete and interval characteristics of the parameters of the regime. Using this method it is possible to perform the analysis of system reliability assessing the control of PS and finding different ways of automatic or operational control allowing PS to move from pre-emergency to acceptable post-emergency state in the event of emergency disturbances.

\section{Methodology}

\subsection{Reliability criteria for power system expansion planning}

The most important criterion for decision-making in operation and in planning of power system development is system reliability and PS viability. Moreover, both balance and regime aspects of system reliability should

Corresponding author: osakalexey@mail.ru 
be provided [5-8]. Planning is carried out at various levels with different time interval, therefore, various aspects of system reliability are considered for different types of planning. When choosing the sufficiency of measures of power grid construction in the development process of power systems, the algorithm given in Fig. 1 is used.

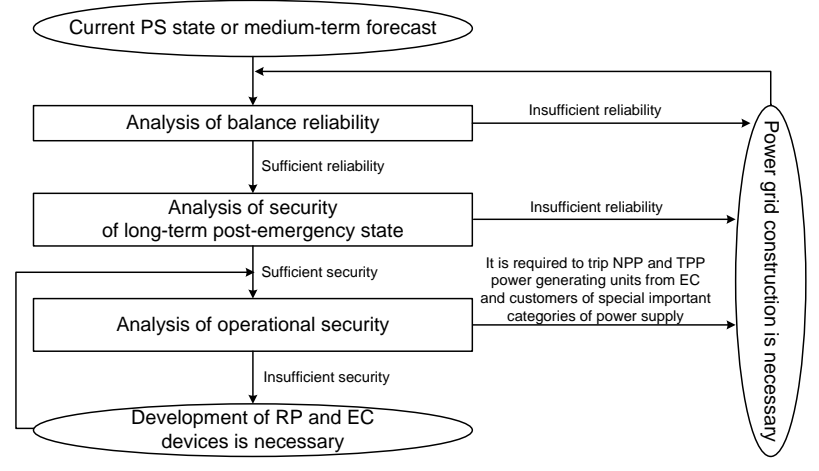

Fig. 1. Algorithm of feasibility studies for measures of power grid construction.

We distinguish two components in assessment of PS security:

Assessment of PS security in the aspect in dynamics process of its transition to postemergency state after emergency disturbances: evaluation of disturbance consequences (including static and dynamic stability analysis), operation of relay protection (RP), emergency control (EC), automatic voltage regulators (AVR), automatic frequency and power control (AFPC), sources of reactive power and on-load tap-changers (OLTC). Changes of generator power are recorded with primary regulation within the primary reserve.

Assessment PS security in the aspect of its recovery process after the potential emergencies and transition from post-emergency to normal state: changes of generators power are recorded with primary, secondary and operational regulation within primary and secondary reserves.

\subsection{Methods and assessment criteria of PS security in the aspect of its recovery process after the potential emergencies and transition from post-emergency to normal state}

At the moment of emergency disturbance by RP and EC operation, partial load shedding (power consumers) is possible as well as output of regime parameters (current, voltage and frequency) beyond permissible values. After $\mathrm{RP}$ and EC response, automatic regulators and regime automatics (RA) together with the operational-dispatcher personnel mobilize hot reserve at power plants. Regime parameters are inserted into permissible limits and power consumers disconnected during the emergency are connected. According to the rules $[10,11]$, it takes 20 minutes to carry out these measures. However, if this time is not enough, the forced regime of power system is introduced and other 20 minutes (totally 40 minutes from the beginning of the emergency) are spent for normalization of the post-emergency regime.

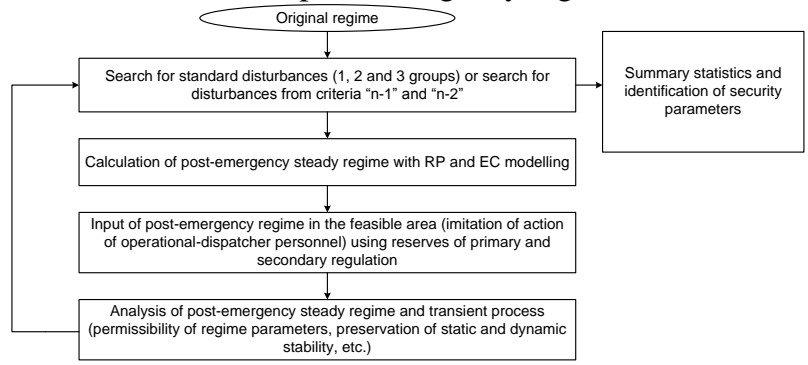

Fig. 2. Algorithm of security analysis during long-term postemergency state of PS

The authors propose to use as one of the PS security criteria the ability of the PS to recover to normal state after an emergency disturbance for 20 (40) minutes, taking into account the operation of the systems of RS, PA, RA and automatic regulators, and further implementation of operational activities by operational dispatch personnel. It is necessary to check if the electricity supply to consumers of the 2nd and higher categories of reliability comply with the requirements for reliability. If it is provided for particular pre-emergency scheme-regime conditions and particular emergency disturbance, it is considered as a sufficient PS security, i.e. it does not require additional power grid construction for improved reliability. If after 20 (40) minutes the restrictions for consumers remain, the volume and probability undersupply of electrical energy are determined, taking into account the subsequent mobilization of all types of reserves.

The authors propose [12] the algorithm for determining the probabilistic characteristics of electrical energy undersupply by iterating over all emergency disturbances and the integration of the probabilistic volume of undersupply (Fig. 2):

1. Original calculating model is developed. Preemergency values of loads and generations in the nodes and state of network elements are determined.

2. Available ranges are specified for regulation of variable regime parameters of static var compensator (SVC), on-load tap-changers (OLTC) of transformers and autotransformers (if automatic or operational tapchange operation is allowed), booster transformers (BT), fixed series compensation (FSC), thyristor-controlled series capacitor (TCSC), as well as generators with active and reactive power. These ranges can be as follows:

- For SVC:

- variable ranges of shunt of capacitor banks (Bmin,

Bmax);

- variable ranges of reactive power ( $Q \min , Q \max )$;

- type of regulation - smooth or discrete (a number of steps or values of changing parameter for each step of control);

- For longitudinal FSC (TCSC) and controlled longitudinal reactors:

- variable ranges of longitudinal resistance (Xmin, Xmax); 
- type of regulation - smooth or discrete (a number of steps or values of changing parameter for each step of control);

- For OLTC of power and phase-variable transformers (autotransformers):

- variable ranges of transformation coefficient (Ktr.min, Ktr.max);

- a number or a step of changing of transformation coefficient;

- For generators:

- permissible values of reactive power ( $Q \min , Q \max$ or in the form of permissible power);

- permissible values of active power (Pmin, Pmax technological minimum and maximum);

- permissible values of changing of active power for 20- and 40-minute range (dPmin.20, dPmax.20, dPmin.40, dPmax.40).

3. Continuous permissible ranges are specified for regime limitations in nodes and branches of the calculating model:

- for voltage (Umin, Umax) and current (Imin, Imax).

4. Limitations are specified for capacity in the controlled sections:

- or in the form of values of maximum available load flow / emergency allowable load flow for concrete conditions of the grid elements (a list of switched on or tripped grid elements);

- or in the form of assignment of vector (trajectory) of weighting for calculation of maximum load flow (MLF) and emergency allowable load flow (EALF) of the concrete controlled section.

5. Controllers of EC device effect are specified:

- or with possible maximal volumes of decrease of load and generation;

- or with EC algorithms stating concrete controlling effects for each stage;

- load category is specified, which can be limited.

6. Possible controlling effects are specified for operational-dispatcher personnel (except specified in Item 2) in the following way:

- enumeration of possible maximal volumes of load shedding and generation of nodes with the help of - operational-dispatcher personnel;

- load category which can be limited.

7. Repair schemes are specified (for a concrete calculation interval: winter/summer etc.):

- or tripping of one grid element (under repair);

- or enumeration of changes of the grid topology (tripping of the equipment under repair, switching on/tripping of the section breakers, etc.), and switching on/tripping of the branches of the calculating model);

- or enumeration of the network topology changes and changes of generation composition (switched on/tripped - of generators and values of active generation power);

- in some cases, if the calculating model does not switch on distribution systems, besides changes of the grid topology and generation, the load redistribution between the nodes is specified (thus, changes of the distribution grid topology is considered including those of the grids of internal power supply of consumers with several supply centers).
8. Enumeration of emergency disturbances caused by long-term grid tripping is specified. Every disturbance is specified by:

- enumeration of tripped grid elements (branches of the calculating model, e.g. TL with tapping is modelled with several nodes and branches);

- probability of concrete emergency disturbance;

- expected time of recovery.

9. Search for emergency disturbances (Item 8) is specified for the original (normal) scheme and for all specified control schemes (Item 7) by turn:

- pre-emergency regime is assessed and changes are overlaid in the control schemes (Item 7, tripping/switching on of branches and changes of generation and load);

- emergency disturbances are searched by turn (Fig. 2) overlaying tripping specified for a concrete disturbance (Item 8);

- EC operation is modelled during estimation of the steady regime (Item 5, in case of its actuation for a concrete disturbance);

- if the iterative process is non-convergence during estimation of the steady regime, it is necessary to model EC (Item 5, using optimization method and method of directed or complete search) to reach post-emergency steady regime;

- if the obtained steady regime has load limitations (load shedding by EC, Item 5) and/or regime parameters go beyond permissible values (Item 3 ) or load flow along the controlled sections exceeds EALF (Item 4, specified or determined by the weighting for achieving postemergency regime), it is necessary to introduce the regime in the allowable area (Items 3 and 4), including the realization of measures available for operationaldispatched personnel (Items 2 and 6) and if possible to recover the load tripped from EC (Item 5);

- if post-emergency regime together with realization of input measures meets all limitations (Items 3 and 4) and if the load tripped from EC but during the input it was possible to recover it, such disturbance does not reduce security;

- if post-emergency regime together with realization of input measures does not meet all limitations (Items 3 and 4 ), it is necessary to fulfil additional adjustment of control able to be applied by operational-dispatcher personnel (Item 6);

- if post-emergency regime together with realization of input measures meets all limitations (Items 3 and 4) but has non-regenerable load (dPlim), it is necessary to calculate electrical energy undersupply (dWlim). Duration of electrical energy undersupply is calculated from the expected time of recovery of the tripped emergency element (Trep), which can be decreased by time of generation mobilization (Tmob), if all permissible values of changes of active power for 40minute range or together with mobilization of cold reserve (limitations on technological maxima and minima) allow completely or partially recovery of preemergency level of load.

10. Integral parameters of electrical power undersupply and damage together with probability are calculated from results of search for all disturbances for all 
calculating models. Moreover, category of consumers included in the limitation list is taken into consideration. 11. If several options of the development of power grid are compared, parameters specified in Item 10 are calculated for each option.

12. The duration of electrical power undersupply $\left(T_{\text {lim.emerg. No. } x x x}\right)$ for a concrete emergency situation is determined from the minimum of mean repair time of the damaged device $\left(T_{\text {rep. emerg. No. xxx }}\right)$ or mean time of mobilization of the generating power $\left(T_{\text {mob. emerg. No. xxx }}\right)$ :

$T_{\text {lim. emerg. No. xxxx }}=\min \left(T_{\text {rep. emerg. No. xxx }}, T_{\text {mob. emerg. No. } x x x}\right)$

Therefore, the value of electrical power undersupply $\left(d W_{\text {lim. emerg. No. } x x x}\right)$ for a concrete emergency situation is determined from the value of power limitation $\left(d P_{l i m}\right.$. emerg. No. $x x x)$ and duration of power undersupply ( $T_{\text {lim. emerg. }}$ No. $x x x)$ :

$\left.d W_{\text {lim. emerg. No. } x x x}=d P_{\text {lim. emerg. No. } x x x} \cdot T_{\text {lim. emerg. No. } x x x}\right)$

The expectation value of the annual monetary loss (ML) from the concrete situation $\left(M L_{\text {ann. emerg. No. xxx }}\right)$ depends on failure flow for a year for concrete damaged equipment during this emergency ( $\left.w_{\text {emerg. No. } x x x}\right)$ :

$$
M L_{\text {ann. emerg. No. } x x x}=w_{\text {emerg. No. } x x x} \cdot d W_{\text {lim. emerg. No. } x x x} \cdot Y_{O . R F}
$$

$M L_{\text {ann. emerg. No. } x x x}=w_{\text {emerg. No. } x x x} \cdot d W_{\text {lim. emerg. No. } x x x} \cdot Y_{\text {O. Reg }}$

Where $Y_{O . R F}$ and $Y_{O . R e g}$ is the energy intensity of the gross domestic product (GDP) for the country as a whole, and the energy intensity of the gross regional product (GRP) for specific subjects of the Federation. For large or particularly important industrial enterprises, the amount of damage is determined individually, but not below the energy intensity.

The expectation value of the annual loss from all emergencies in the power system associated with the electrical power undersupply will be determined from the arithmetical sum of expectation values of annual losses from concrete emergencies $\left(M L_{\text {ann. emerg. No. xxx }}\right)$ :

$$
M L_{\text {ann.emerg }}=\sum_{i=1}^{n} M L_{\text {ann.emerg №i }}
$$

Besides the loss of the expected electrical power undersupply, it is necessary to take into account a probable loss from unforeseen tripping of consumers or generation. It is suggested to consider this loss only in those cases and volumes which are stipulated in the agreements (agreements of power supply or technological connection). If these aspects are not stipulated in the agreement, the loss is not considered. The maximum allowed time of electrical power undersupply for concrete consumers should correspond to agreement conditions and category of consumers (according to the decrees of the Russian Federation Government).

\subsection{PS steady state calculation with taking into account the discrete and interval characteristics of the regime parameters}

The solution of the problem of paragraph 9 is proposed to perform by PS steady state calculation calculating taking into account the discrete and interval characteristics of the regime parameters [13-14].

In general, the balanced steady state parameters as well as their functions should be as close as possible to values $V \rightarrow £$, these values are defined as discrete characteristics of the regimes parameters. They can be measurements, planned values of regimes parameters, forecast values, etc. Elements of $v_{i} \in V$ are characterized by two parameters $\left\{€_{i}, \epsilon_{i}\right\}$. In the case of measurement, $\oint_{i}$ means the dispersion of $€_{i}$, in other cases, it may be the permissible deviations of $v_{i}$ from $€_{i}$, specified as a percentage.

In addition, the $G$ regimes parameters should be in some intervals of their $G \rightarrow[\underline{G}, \bar{G}]$ values, if possible. Accordingly, each interval variable $g_{i}$ is characterized by four parameters $\left\{\underline{g}_{j}, \underline{\sigma}_{j}, \bar{g}_{j}, \bar{\sigma}_{j}\right\}$, where $\underline{g}_{j}, \bar{g}_{j}-$ upper and lower interval limits; $\underline{\sigma}_{j}$, $\bar{\sigma}_{j}-$ boundary dispersions. From $\underline{\sigma}_{j}, \bar{\sigma}_{j}=0$ it follows that $\underline{g}_{j} \leq g_{j} \leq \bar{g}_{j}$. The control range of controlled items (see Item 2), security restrictions (see Item 3-4), the volume control actions (see Item 5-6) are specified using these interval limits.

The current load and generation values, as well as various expected (recommended, optimal) values of voltages, generations and loads of nodes, currents and flows through the branches can be set either by discrete parameters of the $V$, or by interval parameters of $G$ with the values of dispersions (relative deviations) selected by taking into account the ranking of the importance of the recommended constraints or the severity of the control actions. By setting the current load and the active power of the generation by the discrete parameters of $V$, in the simulation of the emergency perturbation first of all will be found the controlling effects, not related to the change in the load and the generation of active power. And only if it is impossible to bring the regimes into the permissible area, the resource controlling effects will be used to reduce the load and change the generation.

Because of the need to rank the role of variables $y_{i}$ in the functioning of the system, it is necessary to take them into account repeatedly in the $G$ with different boundary characteristics and percentage rates $\left(\underline{\sigma}_{i j}, \bar{\sigma}_{i j}\right)$. Accordingly, the wider the range, the lower the percentage rates. The possibility of simultaneous assignments to the same parameter regime of restrictions in the form of discrete values of the expected values of $V$ and several interval ranges $G$ with different values of variance (percentage variance) makes it quite flexible to influence the results of the calculation of the variation of the controlling effects. Using this method, it is possible to perform the analysis of PS security, assessing the control of PS and finding different ways of automatic or operational control allowing PS to move from pre- 
emergency to acceptable post-emergency mode in the event of emergency disturbances.

To characterize the proximity measure of $V \rightarrow ほ$, $G \rightarrow[\underline{G}, \bar{G}]$, the weighted least squares function is commonly used:

$$
f=\sum_{i}^{n} a_{i}^{2}+\sum_{i}^{m} b_{i}^{2}
$$

where $a_{i}=\frac{v_{i}-£_{i}}{\oint_{i}} \cdot k_{v i}$;

$$
b_{i}=\frac{g_{i}-g_{i}^{*}}{\sigma_{i}^{*}} \cdot k_{g i} \cdot k_{i} \text {; }
$$

The coefficients $k_{v i}, k_{g i}$ take into account the regulating effect of interval variables on the variables $v_{i}$ и $\mathrm{g}_{\mathrm{i}}$. If there is a regulating effect of some interval variable $\mathrm{g}_{\mathrm{j}}$ on another variable, for example, $v_{i}$, it exists if the variable $g_{j}$ does not reach one of the boundaries. Therefore $k_{v i_{g i}}=1-k_{j}$.

In the accepted approach, $0 \leq d\left(g_{i}\right) \leq 500$ depends on the distance of $g_{i}$ from the interval boundaries, on the value of $f$ and on the course of the computational process.

$$
\begin{gathered}
g_{i} \leq \underline{g}_{i} \rightarrow\left\{k_{i}=1 ; g_{i}^{*}=g_{i} ; \sigma_{i}^{*}=\underline{\sigma}_{i}\right\} ; \\
g_{i} \geq \bar{g}_{i} \rightarrow\left\{k_{i}=1 ; g_{i}^{*}=\bar{g}_{i} ; \sigma_{i}^{*}=\bar{\sigma}_{i}\right\} ; \\
\underline{g}_{i}<g_{i}<\bar{g}_{i} \rightarrow\left\{\begin{array}{l}
g^{*}=1 ; g_{i}^{*}=g_{i} ; \sigma_{i}^{*}=\underline{\sigma}_{i} \cdot \alpha+\bar{\sigma} \cdot(1-\alpha) ; \\
0<\alpha<1 ; \\
k_{i}=\frac{1}{1+d\left(g_{i}\right)}
\end{array}\right\} ;
\end{gathered}
$$

The steady state of PS is usually described by the equations of balance of currents or powers in the grid nodes and in vector form:

$$
W(X, Y)=0
$$

here, the vector $Y$ is a vector of independent parameters of the regime, which at the stage of solving the formula (2) take specific values. $X$ is the vector of dependent parameters on the vector $Y$. This implicit dependency is indicated by:

$$
X=X(Y) \text {. }
$$

The solution of the problem is reduced to finding such a vector $Y$, at which:

$$
\begin{gathered}
\min f(Y) \\
\text { and } \\
w(X, Y)=0 .
\end{gathered}
$$

We'll solve the problem iteratively. Denote $Y_{k+1}=Y_{k}+Z$. Linearize $f$ at the point $Y_{k}$ :

$$
\begin{aligned}
& a_{i}\left(Y_{k+1}\right)=a_{i}\left(Y_{k}\right)+Z \frac{\partial a_{i}}{\partial y_{k}} \\
& b_{i}\left(Y_{k+1}\right)=b_{i}\left(Y_{k}\right)+Z \frac{\partial b_{i}}{\partial y_{k}} .
\end{aligned}
$$

Substituting these expressions in (1), we obtain a quadratic form $f(Z)$, which has a minimum at $\frac{\partial f}{\partial z}=0:$

$$
\frac{\partial f}{\partial z}=A \cdot Z+B=0
$$

The matrix $A$ is usually non-degenerate, so the system of equations (3) has a solution. The nondegeneracy of the matrix $A$ follows from the fact that all elements of the $Y$ vector belong to $V$ or $G$, and the elements of $Y$ are independent by definition. Therefore, the rank of $A$ is equal to the rank of $Y$. The system of equations (3) always has a solution, even if $V=\varnothing$. It is for this reason $b_{i}$ accounted for on a whole range $g_{i}$.

In the next step, we are looking for a solution to the system:

$$
W\left(X_{k}+d X_{k}, Y_{k}+Z \cdot t\right), t=0
$$

The solution of the system of equations (4) exists if the following criterion is satisfied:

$$
\begin{gathered}
\text { suppose } \Delta W=W\left(X_{k}, Y_{k+1}\right), \\
\Delta X=-\Delta W \frac{\partial W\left(X_{k}, Y_{k+1}\right)^{-1}}{\partial X_{k}} \\
k=2 \sqrt{\left\|\Delta X^{\mathrm{T}} \frac{\partial^{2} W}{\partial X^{2}} \Delta X\right\| /\|\Delta W\|} .
\end{gathered}
$$

For $\mathrm{k}<1$, the solution of the system (4) usually exists. This criterion works well if we are close to the boundary of the limit on the existence of the solution. A more reliable criterion $k<2 / 3$. Therefore, if $k>2 / 3$ choose $t=\frac{2}{3} / k$ and return to the beginning of balancing. It should be noted that the balancing procedure takes $2-3 \%$ of the total time of the task.

Given the balancing $\tilde{Z}=Z \cdot t$ define $f\left(Y_{k}+\tilde{Z} \cdot t\right)$ as a quadratic polynomial of $t$ :

$$
f\left(Y_{k}+\tilde{Z} \cdot t\right)=a_{0}+a_{1} \cdot t+a_{2} \cdot t^{2} .
$$

This polynomial has a minimum at $t=-\frac{a_{1}}{2 a_{2}}$.

If $t<1$ get a new $\tilde{\tilde{Z}}=\tilde{Z} \cdot t$ and return to the step of balancing steady state. 
If $t>1$, then take $t=1$ and go to the next step of the solution of the problem.

The calculation process is terminated when the specified accuracy of minimization is achieved:

$$
f\left(Y_{k}\right)-f\left(Y_{k+1}\right)<\xi \text {. }
$$

\section{Conclusion}

This paper presents methods for assessment of system reliability in operation and during planning of power system development. The method proposed contains general description of the step-by-step algorithm with the help of which it is possible to compare options and scenarios of power system development and to assess the level of reliability control for validation of decision making.

The most significant assessment of security is the evaluation of losses during long power limitation of consumers (over 20 and 40 minutes). The algorithm proposed allows the calculation of probable losses for comparative analysis of different options of PS development.

In comparison with the previously published paper [12], in this paper it was possible to adapt the known method of PS steady state calculation [14] by taking into account discrete and interval characteristics of the parameters of the regimes for the analysis of the PS security. Also the wording of the aspects of PS security is refined.

The work was carried out within the project III.17.4.2 (No. AAAA-A17-117030310438-1) of the fundamental research program of the Siberian Branch of the Russian Academy of Sciences.

\section{References}

1. N.I. Voropai, D.N. Efimov et al., "Smart Technologies in Emergency Control of Russia's Unified Energy System", Smart Grid, IEEE Transactions on. 2013. Vol. 4, no. 3. P. 1732-1740.

2. N.I. Voropai, A.B. Osak, "Power systems of the future", Energy Policy. No. 5. 2014. P. 60-63. (in Russian)

3. Eds. N.I. Voropai, "Reliability of energy systems (Collection of recommended terms)", Moscow: "Energia", 2007. 192 p. (in Russian)

4. "Reliability concepts in bulk electric power systems", New York: North American Electric Reliability Council, 1985. 96 p.

5. N. Balu, T. Bertram, A, Bose et al., "On-line power system security analysis", Proceedings of the IEEE. 1992, Vol. 80, No. 2. P. 262-280.

6. R.J. Marceau, J. Endrenyi, R. Allan et al., "Power system security assessment: A position paper", Electra. 1997, No. 175. P. 49-77.

7. Ed. N.A. Manov, "Methods and models for studying power system reliability", Syktyvkar: Komi SC UrB RAS. 2010. 292 p. (in Russian)
8. Eds. N.I. Voropai, G.F. Kovalev, "Concept of reliability control in electric power engineering", M.: LLC PH "Energy". 2013. 304 p. (in Russian)

9. "Guidelines for stability of power systems", approved by Order No. 277 of 30.06.2016 of Russian Ministry of Energy. (in Russian)

10. “STO 59012820.29.240.007-2008. Rules for prevention of development and disturbance liquidation of the normal state of electrical annex of power systems". Standard JSC "SO UPS". (in Russian)

11. "STO 59012820.27.010.002-2016. Rules of transition to operation in forced regime in controlled sections of the dispatcher center JSC SO UPS", Standard JSC "SO UPS". (in Russian)

12. A. Osak, D. Panasetsky, E. Buzina, Analysis of the security during power system expansion planning // E3S Web Conf. Volume 25. 2017. Article Number 03004. DOI: $10.1051 / \mathrm{e} 3$ sconf/20172503004

13. A.B. Osak, A.I. Shalaginov, D.A. Panasetsky, E.Ya. Buzina, "Method for monitoring of PS state and Assessment of regime reliability from criterion of PS control", Methodic problems of studies of reliability of large power systems / Eds. N.I. Voropai, Yu.Ya. Chukreev. - Syktyvkar: LLC "Komi Republican Printing House", 2016, p. 337346. (in Russian)

14. A.B. Osak, E.Ya. Buzina, "Method and Software for Identifying the Parameters of a Power District". Bulletin of the South Ural State University. Ser. Power Engineering, 2018, vol. 18, no. 1, pp. 53-60. (in Russian) 